\title{
Fuzzy Logic Reliability Centered Maintenance
}

\author{
Felecia $^{1^{*}}$
}

\begin{abstract}
Reliability Centered Maintenence (RCM) is a systematic maintenence strategy based on system reliability. Application of RCM process will not always come out with a binary output of "yes" and "no". Most of the time they are not supported with available detail information to calculate system reliability. The fuzzy logic method attempts to eliminate the uncertainty by providing "truth" in different degrees.Data and responses from maintenance department will be processed using the two methods (reliability centered maintenance and fuzzy logic) to design maintenance strategy for the company. The results of the fuzzy logic RCM application are maintenance strategy which fit with current and future condition.
\end{abstract}

Keywords: Reliability centered maintenance, fuzzy logic.

\section{Introduction}

Maintenence is an act to keep equipment in existence or continuance and also to keep it in appropriate condition (Higgis and Mobley [1]). Equipment maintenence has been changed in the last century, and this is due to increase in physical assets, more complex equipment system, and greater emphasis on safety and environment. Maintenence function expectation has also been growing for the last century. The first period during 1930-1950 equipment was simple, over designed and reliable, so breakdown maintenence is more applicable. Second period during 1950-1980 there were increased demand in manufactured goods but limited supply of skilled labour. Preventive maintenence were developed during this second period. Third period 1980 - now, there were mass production, mass transportation, industrial automation and equipment complexity (Moubray [2]). RCM was developed within aircraft industry in third period but also adapted to other industries (Rausand and Vatn[3]).

Reliability Centered Maintenance (RCM) is a wellstructured, logical decision process used to identify the policies needed to manage failure modes that could cause the functional failure of any physical item in a given operating context (Moubray [2]). The methodology is based on the assumption that the inherent reliability of the equipment is a function of design and the built quality. RCM concentrates on the preservation of function instead of focusing on the hardware. RCM treats components differently in terms of relative importance according to the correlation between the equipment and the system function.

1 Faculty of Industrial Technology, Industrial Engineering Department, Petra Christian University, Jl. Siwalankerto 121-131 Surabaya 60238, Indonesia. Email: felecia@peter.petra.ac.id

*Corresponding author
The goal is to develop a maintenence strategy fit with current and future condition (Tomlingson [4]). Beside that good maintenence strategy also needed to optimized long-run cost and short-term budgets (Gosavi [5]). These strategies are based on the risks posed by thefailures. Each failure mode is handled on its merits by addressing its potential consequences and by the type of failure (Narayan [6]).

Application of RCM process will not always come out with a binary output of "yes" and "no". Most of the time they are not supported with available detail information to calculate system reliability.

The fuzzy logic method attempts to eliminate the uncertainty by providing "truth" in different degrees. According to Zadeh [7], a fuzzy set is a class of objects with a continuum of grades of membership. It has a membership function which assigns to each object a grade of membership between zero and one. Fuzzy logic RCM application will use information from maintenance department to design maintenance strategy which fit current and future condition.

\section{Methods}

\section{Reliability Centered Maintenance (RCM)}

$\mathrm{RCM}$ is a process used to determine the maintenence requirements of any physical assets in its operating contex (Ahmadi et al. [8]). RCM has been used in the airline industry since 1970s and has been developed into non-aviation industry. There are seven basic RCM questions: (1) What are the functions of the asset in its present operating context? (2) In what ways does it fail to fulfill its functions? (3) What causes each functional failure? (4) What happens when each failure occurs? (5) In what way does each failure matter? (6) What can be done to predict or prevent each failure? (7) What should be done if a suitable proactive task cannot be found? 


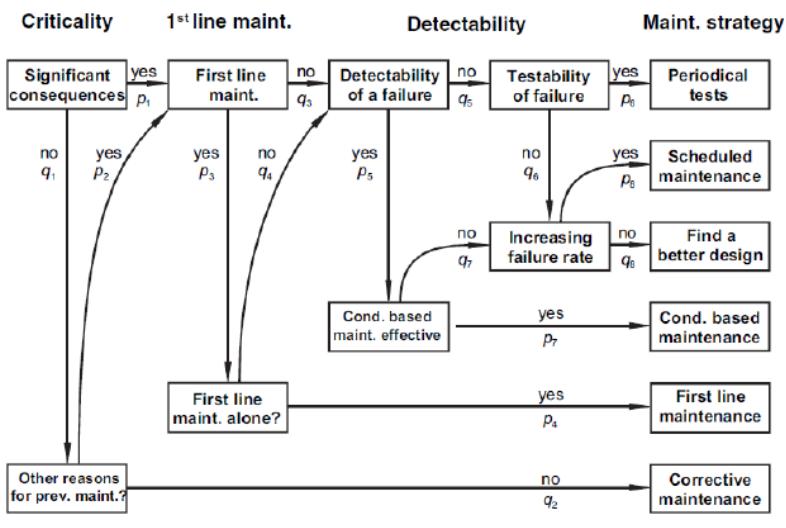

Figure 1. Example of RCM decission diagram

These seven questions in can be formed as RCM decission diagram as in Figure 1 will facilitate the choice of optimum maintenence strategy.

The RCM analysis may be carried out as a sequence of activities or steps, including study preparation, system selection and identification, functional failure analysis, critical item selection (significant item selection), data collection and analysis, Failure Mode Effect and Criticality Analysis (FMECA), selection of maintenance actions,determination of maintenance intervals, preventive maintenance comparison analysis, treatment of non-critical items, implementation and in-service data collection and updating (Ahmadi et al. [8], Rousand [9]).

The available failure management strategies offered by RCM consist of specific scheduled maintenance tasks selected on the basis of the actual reliability characteristics of the equipment which they are designed to protect, and they are performed at fixed, predetermined intervals. The objective of these tasks is to prevent deterioration of the inherent safety and reliability levels of the system.

General approaches to reliability and risk management, include the identification of hazards, the objects that could be harmed, and controls for reducing the frequency or consequence of unwanted events. The most important part of risk analysis is risk identification. Only those risks that have been identified can be managed in a systematic and conscious way (Moubray [2]). The consideration of risk as a criterion for selecting the maintenance policy is crucial.

RCM encompasses the well-knownFailure Mode and Effects Analysis (FMEA) methodology. FMEA starts with makingan end item breakdown into significant functional or hardware items, identifyingsystem boundaries, and showing the relationships of components or functions with each other. The FMEA defines the significant item(s) and establishes the cause- and-effect relationships among its/their function(s), functional failures(s) and failure mode(s), the end effects of the functional failures, and the consequences of failure(s), which aims at determining possible system states under the assumption of the presence of certain failures. This includes the determination of local effects, system effects, end effects and the failure detection methods (Conachey and Montgomery [10]).

When analizing the safety, operational, and economic consequences of failures, one major challenge is assessment of the operational risk of failures, and its associated costs. This is due to the long list of uncertainties related to the large amount of influencing factors, the inadequacy of service information, and difficulties in understanding the influences of the different factors. The difficulty is that, during the initial maintenance programme development, there is a lack of data (Tomlingson [4]).

Data required for reliability maintenence programme are equipment information, failure information, maintenance information, cost data, condition monitoring data, and spare parts inventory information (Tang et al.[11]). Equipment information, detailed and accurate equipment information enabling each component item to beseparately identified. Hierarchy and boundary definitions of equipment are necessary. Operating mode, such as active or stand by are identified. Example of the information include: the unique identification number, classification, location, installation, operations situation.

Failure information, adequate information about the failures, e.g., failure mode, failure cause, failure consequences, failure description, severity class. The failure modes should be accurately defined. Maintenance information, accurate information relates to downtime, repair time and restore time. It contains information about the actual preventive and corrective maintenance being carried out. Maintenance type, such as corrective and preventive replacement should be identified. Cost data are thecost of failure such as the penalty cost of system outage (e.g. loss of production). Cost ofcorrective repair effort, cost of preventive maintenance, and associated spares and other maintenance cost.

Condition monitoring data, measurements related to the health condition (indicator) of the asset. Condition monitoring data are very versatile. It can be vibration data, oil analysis data, acoustic data, temperature, pressure, moisture, humidity, weather or environment data. Spare parts inventory information, information about spare parts storage such as stock level, holding cost, lead time, spares demands. 
When the real data is not available, experts experience can still be used as a valuable source of information to estimate the required data. This is not in contradiction with the conservative approach when the real data is not available. The risk can be assessed either quantitatively or qualitatively. For most RCM analyses, a simple risk matrix is used the risk (Guo et al. [12]).

RCM itself has three approachs: qualitative, probabilistic, and fuzzy approach. Qualitative has its draw backs due to concensus requirements on each question and does not provide a ranking of strategies. Probabilistic approach interprets the degree of an expert's belief as the mean value for a decission. Fuzzy aproach allows subjective assessment and expert experience as a fuzzy input variable.

\section{Dampster-Shaffer Theory}

Dampster-Shaffer Theory (DST), which also known as belief function theory, is a generalization of Bayesian theory for subjective probabilities. DST introduced in the field of reliability in the early 1990s. It is based on a scenario that contains the system with all hypotheses, pieces of evidence and data sources.

The hypotheses represent all possible states in the system. It is required that all hypotheses are unique, not overlappingand mutually exclusive. Pieces of evidence are symtoms or events that occur or may occur in the system. Data sources are persons or organizations that provide information for a scenario. Insafety and reliability engineering, data sources areusually the results of empirical studies or they are experts, who give subjective quantifiable statements (Rakowsky and Gocht [13], Rakowsky [14]).

By applying the basic assignment function, several evidential functions can be created. A belief measureis given by the function bel: $2^{\Omega} \rightarrow[0,1]$. There is

$\operatorname{bel}(\boldsymbol{A})=\sum_{B \subseteq A ; B \neq \varnothing} m(\boldsymbol{B})$

The counterpart of $b e l$ is the plausibility measure $p l$ : $2^{\Omega} \rightarrow[0,1]$ with

$p l(\boldsymbol{A})=\sum_{B \cap A \neq \varnothing} m(\boldsymbol{B})$

Damster Shafer rule of compbinationwhich allows that the basic assignmentsare combined.

$m_{1,2}(\boldsymbol{Z})=\frac{\sum_{A \cap B=Z \neq \emptyset} m_{1}(\boldsymbol{A}) m_{2}(\boldsymbol{B})}{1-\sum_{A \cap B=Z \neq \emptyset} m_{1}(\boldsymbol{A}) m_{2}(\boldsymbol{B})}$

with $\boldsymbol{A}, \boldsymbol{B}, \boldsymbol{Z} \Omega$. The numerator represents the accumulated evidence for the sets $\boldsymbol{A}$ and $\boldsymbol{B}$, which supports the hypothesis $\boldsymbol{Z}$, and the denominator sum quantifies the amount of conflict between the two sets.

There are several terms used in DST, basic assignment (m), belief (bel), and plausibility (pl).

Figure 2 shows a graphical representation of the above-defined measures belief and plausibility. The difference $p l(\boldsymbol{A})-\operatorname{bel}(\boldsymbol{A})$ describes the evidential interval range, which represents the uncertainty concerning the set $\boldsymbol{A}$.

\section{Fuzzy Logic}

Fuzzy logic is developed from the theory of fuzzy set by Zadeh [7]. Fuzzy logic has been extended to handle the concept of partial truth, where the truth value may range between completely true and completely false. Fuzzy set used words and not number to represent certain condition like"no", "few", "some", "many", "yes". Membership function in fuzzy logic represents the degree of truth in the real world and this will need a good formulation. There are a few type of fuzzy logic membership function, but triangular dan trapezoidal is the most commondly used (Klir [15], Wang [16].

Triangular membership function formed by three parameters $(a, b, c)$ which $(a<b<c)$. Figure 3 .

Triangle $(\mathrm{x} ; \mathrm{a}, \mathrm{b}, \mathrm{c})=\left\{\begin{array}{rc}0, & x \leq a \\ \frac{x-a}{b-a}, & a \leq x \leq b \\ \frac{c-x}{c-b}, & b \leq x \leq c \\ 0, & 0 \leq x\end{array}\right.$

Trapeziodal membership function formed by four parameters (a,b,c,d) which (a,b,c,d). Figure 4.

Trapezoidal $(x ; a, b, c, d)=\left\{\begin{array}{rc}0, & x \leq a \\ \frac{x-a}{b-a}, & a \leq x \leq b \\ 1, & b \leq x \leq c \\ \frac{d-x}{d-c}, & c \leq x \leq d \\ 0, & d \leq x\end{array}\right.$

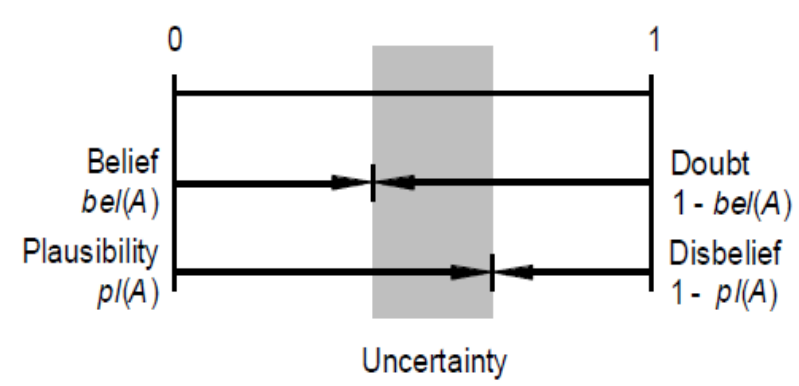

Figure 2. Measure of belief and plausability 


\section{Fuzzy Logic Process}

Fuzzy logic has three steps in the process: fuzzification, rule evaluation, and defuzzification. Fuzzification is a process to transform crips values into grades of membership for linguistic terms of fuzzy set (Wang[16]). Figure 5. The membership function is used to associate a grade to each linguistic term. Rule evaluation is a process to to find fuzzy output from fuzzy input based on if_then rules. Figure 6 . Defuzzification is a process to transform fuzzy output into crips output using output membership function. Figure 7.

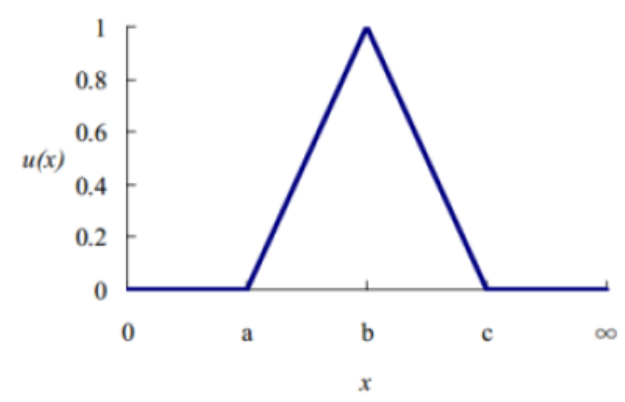

Figure 3. Triangular membership function

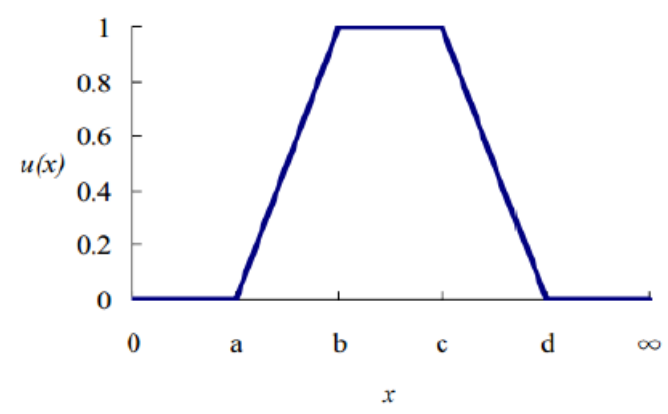

Figure 4.Trapeziodal membership function

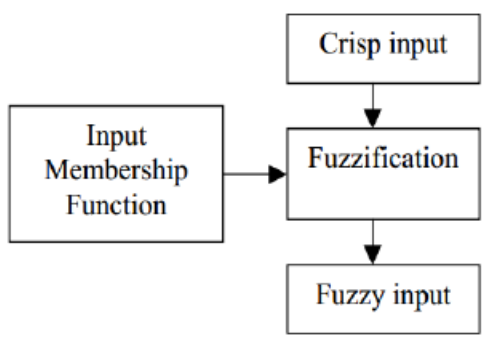

Figure 5. Fuzzification process

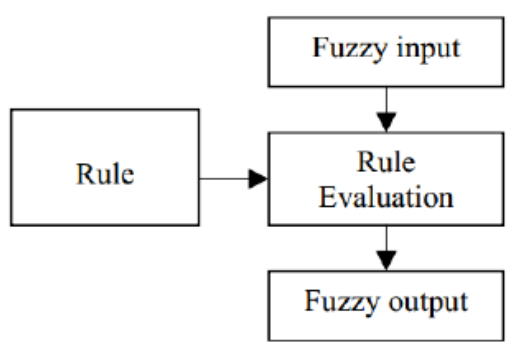

Figure 6. Rule evaluataion process

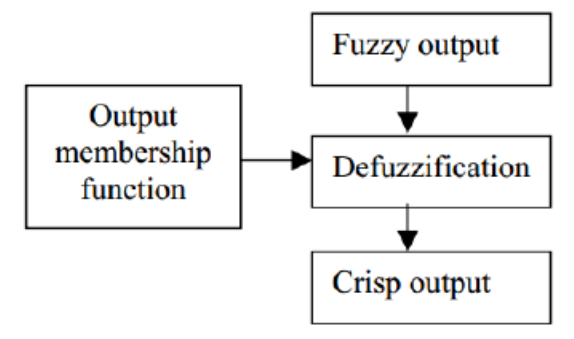

Figure 7. Defuzzification process

\section{Results and Discussion}

\section{Failure Identification}

Application of fuzzy logic RCM is held in an international thread manufacturing company in Surabaya, Indonesia. There three types of machine used in the process of thread manufacturing: doubling, twisting, and winding machines. Each machine is identified for component failure which caused the production stopped. This company used four types of maintenance: preventive maintenance, corrective maintenance, condition based maintenance, and breakdown maintenance.

Preventive maintenence is done for component which have significants effects for the production. Corrective maintenence is done when failure pattern can be recognise. Condition based maintenence can be done only when failure can detected. Breakdown maintenence is done when component failure has no significant effect, no failure pattern, and also can not be detected. RCM decision diagram is build based on maintenance system available in the company. Figure 8.

Decission to use certain type of maintenance for each failure most of the time is based on experience of the maintenance staff in the company. Most of the time there is no absolute "yes" or "no" for every question asked in this RCM decision diagram. Fuzzy logic will be used to solve the problem by giving degree of truth gradually.

Fuzzy inference model requires: fuzzy input output variable, membership function and if_then rules. Fuzzy input variables consist of: failure effect (Figure 9), failure pattern (Figure 10), failure symptom (Figure 11). Input membership function is build by creating linguistic label for each input variable. Linguistic label will help maintenence staff to differentiate and rank the degree for each input variable. The label for failure effect started from "very low", "low", "medium", "high" and "very high". The label for failure patern and failure symptom started from "very clear", "clear", "medium", "not clear" and "very not clear". 
Each lebel of membership function has overlap section with other label, and thsese are the effect of the fuzzy input varible. Example score of 4 for failure sympton can be considered as "clear" and "medium". There are 72 rules based on combination of the input variables. These rules used to decide type of maintenance based on fuzzy output variables. Since there are four type of maintenance used in the company, output variables also devided evenly to accommodate this.Preventive maintenance, between 0 and 2.5.

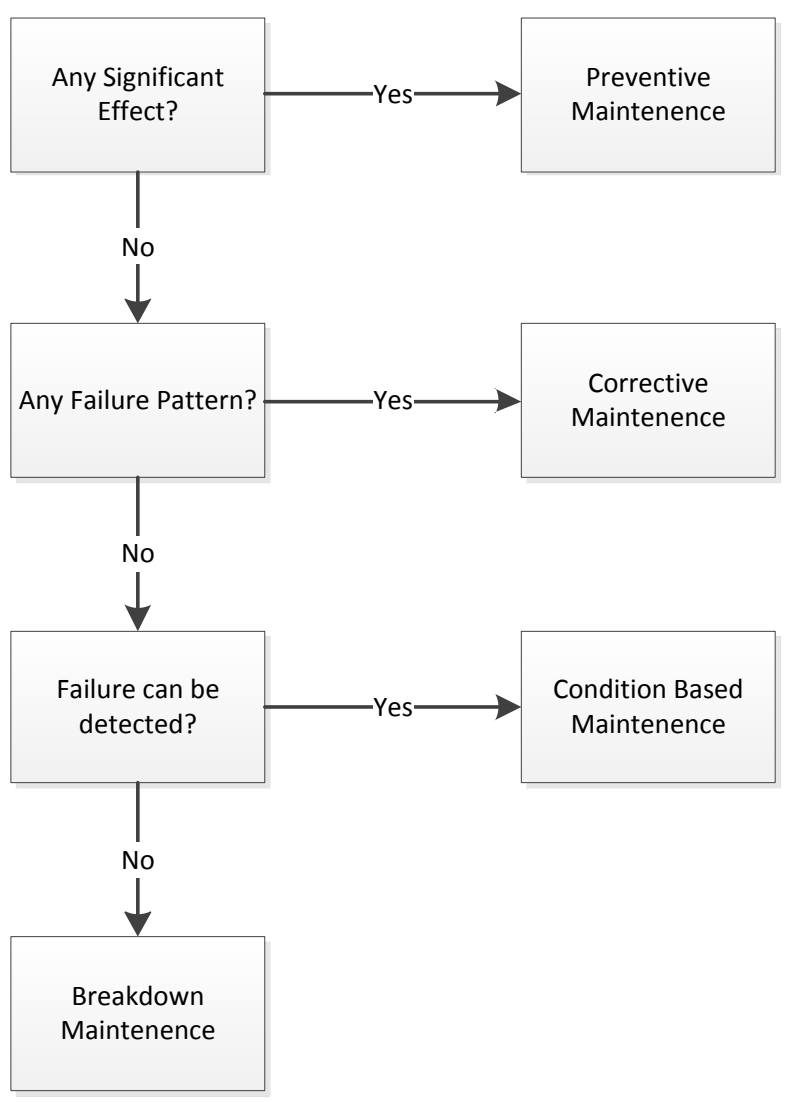

Figure 8. RCM decission diagram

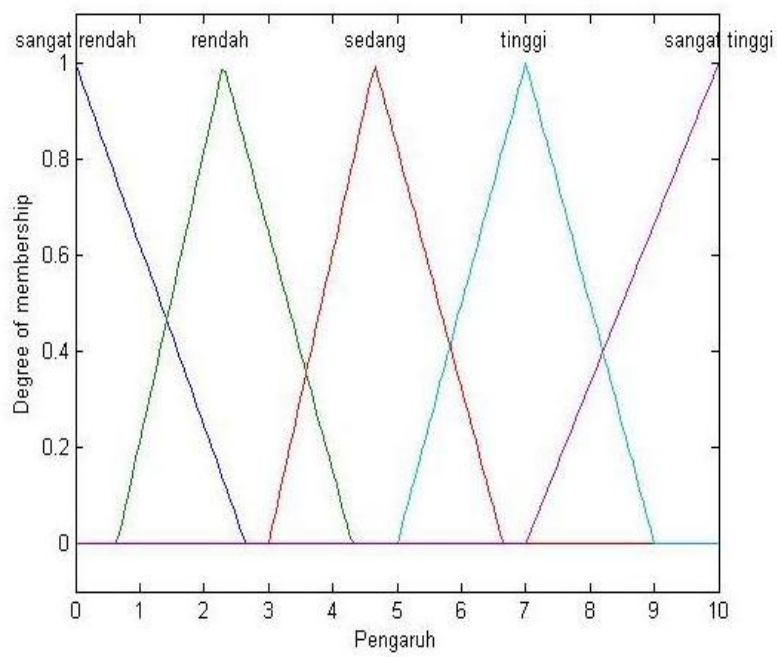

Figure 9. Membership function of failure effect

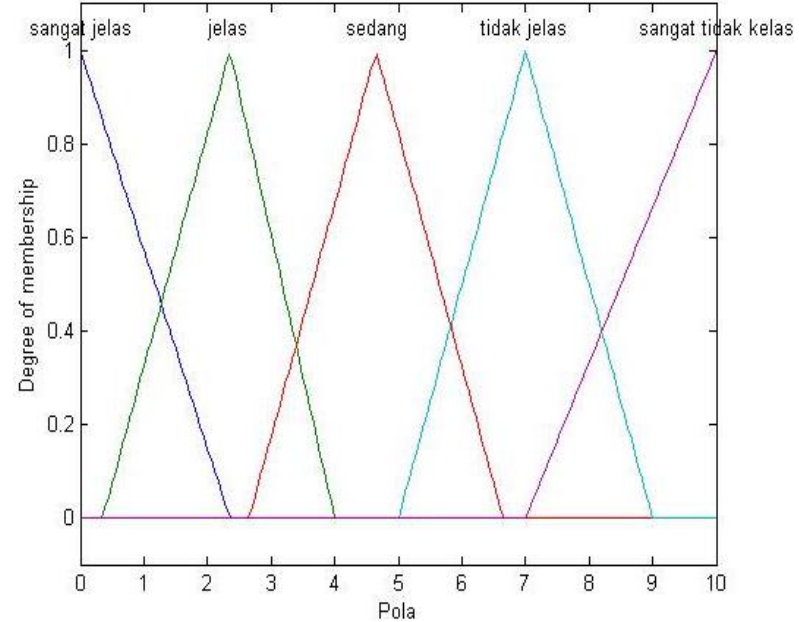

Figure10. Membership function of failure pattern

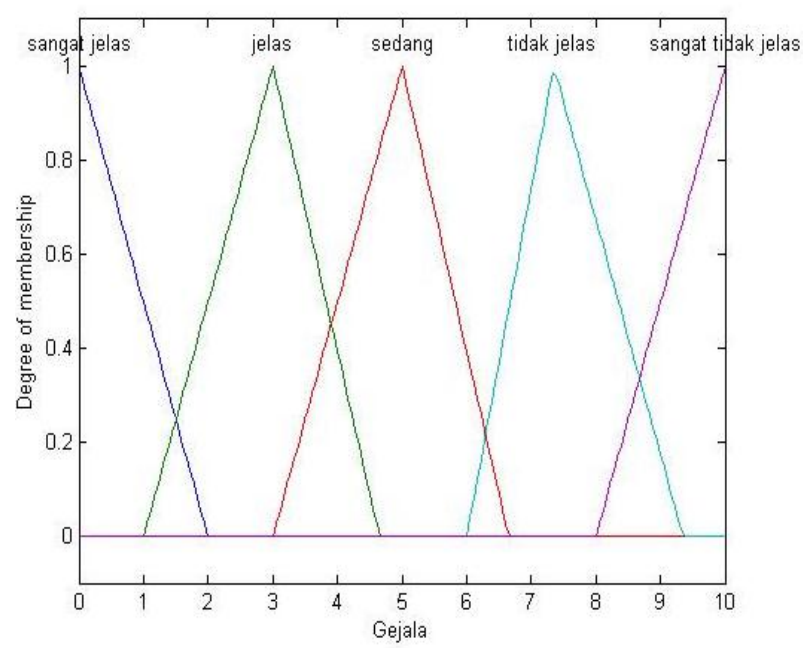

Figure 11. Membership function of failure symptom

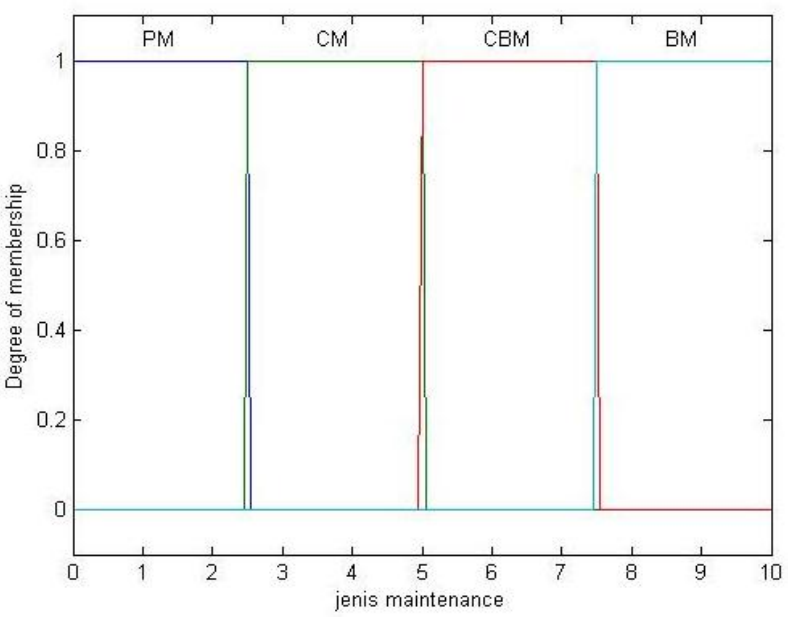

Figure 11. Membership function of maintenance type

Corrective maintenance, between 2.5 and 5. Condition Based Maintenance, between 5 and 7.5. Breakdown Maintenance, between 7.5 and 10. 
Table 1. Result of Fuzzy RCM

\begin{tabular}{|c|c|c|c|c|c|}
\hline Machine Component & Effect ] & Patterm & ymptc & Maint & enence \\
\hline Doubling Wire & 2.33 & 4.50 & 4.68 & 3.75 & $\mathrm{CM}$ \\
\hline Automatic & & & & & \\
\hline Roll & 2.33 & 9035 & 5.00 & 6.25 & $\mathrm{CBM}$ \\
\hline Gear & 3.00 & 7035 & 2.68 & 6.25 & CBM \\
\hline Eyelet & 2.33 & 4.50 & 4.83 & 3.75 & $\mathrm{CM}$ \\
\hline Ear & 2.33 & 4.50 & 4.83 & 3.75 & $\mathrm{CM}$ \\
\hline Vanbelt & 10.00 & 2.35 & 1.35 & 1.25 & PM \\
\hline Twisting Wire & 4.50 & 9.35 & 7.35 & 8.75 & $\mathrm{BM}$ \\
\hline Yarn Guide & 1.35 & 8.65 & 7.18 & 8.75 & $\mathrm{BM}$ \\
\hline Handle & 2.68 & 4.50 & 4.18 & 3.75 & $\mathrm{CM}$ \\
\hline Pig tail & 2.33 & 7.35 & 7.83 & 8.75 & BM \\
\hline Tire & 4.68 & 1.65 & 3.18 & 3.75 & $\mathrm{CM}$ \\
\hline $\begin{array}{l}\text { Tensor } \\
\text { socket }\end{array}$ & 2.33 & 7.35 & 1.18 & 6.25 & $\mathrm{CBM}$ \\
\hline Winding Scisor & 2.00 & 4.50 & 5.00 & 3.75 & $\mathrm{CM}$ \\
\hline Vanbelt & 10.00 & 2.35 & 1.35 & 1.25 & PM \\
\hline Ceramic & 2.33 & 7.35 & 7.33 & 8.75 & $\mathrm{BM}$ \\
\hline Seal & 10.00 & 2.18 & 1.33 & 1.25 & PM \\
\hline $\mathrm{PM}$ & ten & & & & \\
\hline Correct & itenc & & & & \\
\hline Condition $B$ & $\operatorname{sed} M c$ & tenc & & & \\
\hline BM : Breakdown $M$ & Iaintenc & ince & & & \\
\hline
\end{tabular}

The result of fuzzy logic RCM application (Table1) in this company shows that preventive maintenance should be used for component that has high failure effect, such as vanbelt and seal. Corective maintenence suitable for wire, eyelet, ear, handle tire and scisor which has failure pattern. While component which failure can be detected should use condition based maintenance. Componen suitable for this strategy are roll, gear and tensor socket. Breakdown maintenance are suitable for component with no significant effect, no failure pattern and it's failure cannot be detected. Breakdown maintenence sould be done for yarn guide, pig tail and ceramic.

\section{Conclusion}

Fuzzy Logic RCM can be applied to help company to maintenance strategy which fit with current and future condition. Company will be able to alocate its resources more efficiently based on the maintenence strategy proposed. Periodic review will be needed to evaluate validity of the maintenence strategy due to changes that might occur in the fuzzy input and the deterioting condition of the machines.

\section{References}

1. Higgis, L.R., and Mobley, R.K., Maintenance Engineering Handbook, 6th ed., McGraw-Hill, 2002.

2. Moubray, J., Reliability-centered Maintenance, $2^{\text {nd }}$ edition, Butterwort-Heinemann: Oxfort, 1997.
3. Rausand, M., and Vatn, J., Realiability Centered Maintenance, Risk and Reliability in Marine Technology, Balkema, Holland, 1998.

4. Tomlingson, P.D., Reliability-centered Maintenence Improves Equipment Health, Journal Mining Engineering, 61(12), 2009, pp.41-49.

5. Gosavi, A., Murray, S.L., Tirumalasetty, V.M., and Shewade, S., A Budget-Sensitive Approach to Scheduling Maintenance in a Total Productive Maintenance (TPM) Program, Engineering Management Journal, 23(3), 2011, pp. 46-56.

6. Narayan, V., Business Performance and Maintenance How are Safety, Quality, Reliability, Productivity and Maintenance Related?, Journal of Quality in Maintenance Engineering, 18(2), 2012, pp. 183-195.

7. Zadeh, L.A., A Simple View of the DampsterShafer Theory of Evidence and its Implication for the Rule of Combination, AI Magazine, 7(2), 1986.

8. Ahmadi, A., Soderholm, P., and Kumar, U., Aircraft Schedule Maintenence Program Development, Journal of Quality in Maintenence Engineering, 16(3), 2010, pp. 229-255.

9. Rousand, M., Reliability Centered Maintenence. Reliability Engineering and System Safety, 60(2), 1998, pp. 121-132.

10. Conachey, R.M., and Montgomery, R.L., Application of Reliability-centered Maintenance Techniques to the Marine Industry, ABS Technical Paper, 2003, pp. 39-60.

11. Tang, T., Banjevic, D., and Jardine, A.K.S., Design of Reliability Knowledge Database Model, Proceedings of the 2008 Industrial Research Conference, Vancouver, Canada, 2008.

12. Guo, L., Gao, J., Yang, J., and Kang, J., Criticality Evaluation of Petro chemical Equipment Based on Fuzzy Comprehensive Evaluation and a BP Neural Network, Journal of Loss Prevention in the Process Industries, 22(4), 2009, pp. 469-476.

13. Rakowsky, U.K., and Gocht, U., Reasoning in Realiability-Centered Maintenence Based on a Dampster-Shafer Approach, IMechE. 222 Part O, Journal of Risk and Reliability, 2008, pp. 605612.

14. Rakowsky, U.K., Fundamentals of The Dampster-Shafer Theory and Its Application to System Safety and Reliability Modelling, RTA \# 3-4, December - Special Issue, 2007, pp. 173-185.

15. Klir, G.J., and Bo Yuan, Fuzzy Sets and Fuzzy Logic, Theory and Applications, Michigan: Prentice Hall International, 1995.

16. Wang, Li Xin, A Course in Fuzzy System and Control, New Jersey: Prentice-Hall International, 1997. 